\title{
Effect of testicular consistency on semen quality of Murrah buffalo breeding bulls
}

\author{
Satendra Kumar Yadav, Pawan Singh and Navav Singh
}

Received: 18 August 2018 / Accepted: 30 November 2018 / Published online: 21 February 2019

(C) Indian Dairy Association (India) 2019

\begin{abstract}
The present study was conducted to find out the relationship between testicular consistency and semen quality of Murrah buffalo breeding bulls. For this study, 64 mature Murrah buffalo breeding bulls, which were in regular semen collection, were selected. Four ejaculates of each bull were evaluated for semen quality attributes viz. ejaculate volume $(\mathrm{EV}, \mathrm{ml})$, mass motility (MM, 0-5), sperm concentration (SPC, million/ml), sperm motility (SM, \%), viability (\%) and sperm abnormality (SA, \%). Testicular consistency was measured by palpation of the testis and categorised as soft, moderate and firm state; the bulls were also grouped on the basis of testicular consistency as soft, moderate and firm group. The results revealed that EV, MM, SPC, SM, sperm viability and SA did not show any significant difference among the groups but better quality semen was obtained in moderate testicular group than firm and soft groups. From this study it can be concluded that the bulls having moderate testicular consistency produced better quality semen, therefore testicular consistency can also be included in routine breeding soundness evaluation of buffalo bulls.
\end{abstract}

Satendra Kumar Yadav

Department of Livestock Production Management,

Post Graduate Institute of Veterinary Education and Research, Jaipur-

302031, Rajasthan, India

Pawan Singh $(\bowtie)$

Livestock Production Management,

ICAR-National Dairy Research Institute, Karnal-132001, Haryana,

India

E-mail: pawansinghdabas@gmail.com

Navav Singh

Department of Livestock Production Management,

Post Graduate Institute of Veterinary Education and Research, Jaipur-

302031, Rajasthan, India
Keywords: Murrah bull, Testicular consistency, Semen quality

\section{Introduction}

Buffalo is the main dairy animal of our country as it contributes $49 \%$ of total milk production (BAHS, 2017) while the huge population (47.15\%) of buffaloes are non-descript (BAHS, 2017) therefore need to upgrade these buffaloes for improvement in her productivity. An artificial insemination with frozen semen of superior bulls has played an important role in up gradation and improvement of productivity. This is also being adopted in buffaloes at faster rate, as a result demand of buffalo frozen semen and bulls have increased. It is important that maximum number of females be served with the quality frozen semen of superior genetic constitution (Singh and Pant, 2000). Therefore, AI with quality frozen semen from genetically superior bulls is the only way through which a sizable population can be covered and genetically improved. The consistency of testis also plays a major role in quality semen production. It refers to the firmness and resilience (springiness) of the testicles and is a good indicator of testicular function and semen quality. Due to change in testicular consistency the quality of semen affected which influences the fertility of bull (Ashwood, 2009). Testicular consistency has not been assessed and associated with semen quality so far in buffalo breeding bulls. The present study was attempted to determine the relationship between testicular consistency and semen quality of Murrah buffalo breeding bulls.

\section{Materials and methods}

The study was conducted on 64 breeding bulls of Murrah buffalo in winter, maintained at different institutes of Haryana and Punjab $v i z$. Artificial Breeding Research Centre (ABRC), ICAR-National Dairy Research Institute (NDRI), Karnal, Haryana; ICAR-Central Institute for Research on Buffalo (CIRB), Hisar, Haryana; Frozen Semen Stations of Haryana Livestock Development Board (HLDB) situated at Jagadhri of Haryana; and Guru Angad Dev Veterinary and Animal Sciences University, Ludhiana, Punjab, India. The bulls were kept in individual bull pen and fed according to standard feeding schedule along with ad lib seasonally available green fodder and free access to fresh drinking water throughout the day with continuous supply. Vaccination, de-worming and 
other herd-health programme were followed as per the standard protocol and made to regular exercise in the bull exerciser day prior to semen collection. The following parameters were taken for the study-

\section{Testicular consistency by manual palpation}

The consistency of bull's ( $\mathrm{n}=64$ ) testes were assessed by manual palpation of testicles and subsequently scored into three different categories on the basis of firmness and resilience of testicles. Firmness is judged by the distance the tissue can be depressed when squeezed and resilience is the natural tendency of the tissue to return to its normal shape after pressing.

Score 1: Soft

Score 2: Moderate

Score 3: Firm/Hard

\section{Ultrasonographic examination of testes}

The parenchyma of testis was scanned with the help of ultrasound machine (KAIXIN KX 2600, Xuzhou Kaixin Electronic Instrument Co. Ltd. China) using linear probe taking the frequency $6.5 \mathrm{MHz}$ in 40 bulls. The probe was placed longitudinally on the dorsal surface of testicles. (Figure-1)

\section{Semen collection and their quality evaluation}

Semen was collected twice in a week from the bulls with the help of artificial vagina (AV) using standard protocol. The collected semen was immediately put into the water bath at $37^{\circ} \mathrm{C}$ and various standard laboratory tests were performed. The quality of fresh semen was assessed in terms of ejaculate volume $(\mathrm{ml})$, mass motility (0-5 scale) and percent sperm motility (Kiani et al., 2014), sperm concentration $($ million $/ \mathrm{ml})$ with the help of improved Neubauers chamber and IMV photometer, percent sperm viability and abnormality using eosin-nigrosine staining. The above motility, viability and abnormality seminal parameters were observed in phase contrast microscope (Nikon Eclipse E600, Tokyo, Japan) equipped with a warm stage $\left(37^{\circ} \mathrm{C}\right)$.

Other seminal attributes viz. sperm concentration per ejaculates (SPCE), total motile sperms per ejaculates (TMSE) and total live sperms per ejaculates (TLSE) were also measured for individual bulls using following formulae-

$\%$ sperm motility x ejaculate volume (ml) x sperm concentration (million $/ \mathrm{ml}$ ) TMSE $=$

100

$\%$ sperm viability $\mathrm{x}$ ejaculate volume $(\mathrm{ml}) \mathrm{x}$ sperm concentration (million $/ \mathrm{ml}$ ) TLSE $=$

100

In present study the bulls ( $\mathrm{n}=64$ ) were divided into three groups on the basis of their testicular consistency viz. Soft, Moderate and Firm/hard.

\section{Statistical analysis}

The data statistically analysed with respect to testicular consistency and semen attributes by one way ANOVA using SPSS version 20 (statistical analysis software) and the means were compared by Duncan multiple range test. The significance level was tested at $95 \%(\mathrm{P} \leq 0.05)$.

\section{Results and discussion}

In present study the semen attributes viz. EV, MM, SPC, SM, viability, SA, SPCE, TMSE and TLSE did not differ significantly $(\mathrm{p}<0.05)$ among the groups, however, the quality of semen observed better i.e. lower SA and higher MM, SPC, SM, viability, SPCE, TMSE and TLSE in group-2 of moderate testicular

Table 1 Effect of testicular consistency on semen quality of Murrah breeding bull (Mean $\pm \mathrm{SE}$ )

\begin{tabular}{lllc}
\hline Parameters & Group 1 Soft $(\mathrm{n}=16)$ & Group 2Moderate $(\mathrm{n}=27)$ & Group 3Firm/Hard(n=21) \\
\hline EV $(\mathrm{ml})$ & $3.37 \pm 0.23$ & $3.28 \pm 0.26$ & $3.40 \pm 0.20$ \\
MM & $3.20 \pm 0.11$ & $3.33 \pm 0.09$ & $3.15 \pm 0.10$ \\
SPC $(\mathrm{m} / \mathrm{ml})$ & $1000.99 \pm 38.99$ & $1137.92 \pm 76.69$ & $1019.79 \pm 87.69$ \\
SM $(\%)$ & $73.70 \pm 2.03$ & $76.13 \pm 1.08$ & $73.12 \pm 2.22$ \\
Viability $(\%)$ & $82.20 \pm 1.34$ & $84.28 \pm 1.04$ & $81.37 \pm 1.73$ \\
SA $(\%)$ & $9.16 \pm 0.96$ & $8.07 \pm 1.23$ & $8.37 \pm 0.95$ \\
SPCE (million) & $3324.41 \pm 236.93$ & $3635.46 \pm 314.96$ & $3328.38 \pm 260.27$ \\
TMSE (million) & $2487.93 \pm 196.80$ & $2800.00 \pm 268.38$ & $2480.23 \pm 210.70$ \\
TLSE(million) & $2751.63 \pm 208.00$ & $3079.92 \pm 280.66$ & $2733.97 \pm 222.90$ \\
\hline
\end{tabular}

$\mathrm{EV}=$ Ejaculate Volume, $\mathrm{MM}=$ Mass Motility, $\mathrm{SPC}=$ Sperm Concentration, $\mathrm{SM}=$ Sperm Motility, $\mathrm{SA}=$ Sperm Abnormality, $\mathrm{SPCE}=$ Sperm Concentration per Ejaculate, TMSE= Total Motile Sperms per Ejaculate, TLSE= Total Live Sperms per Ejaculate. 


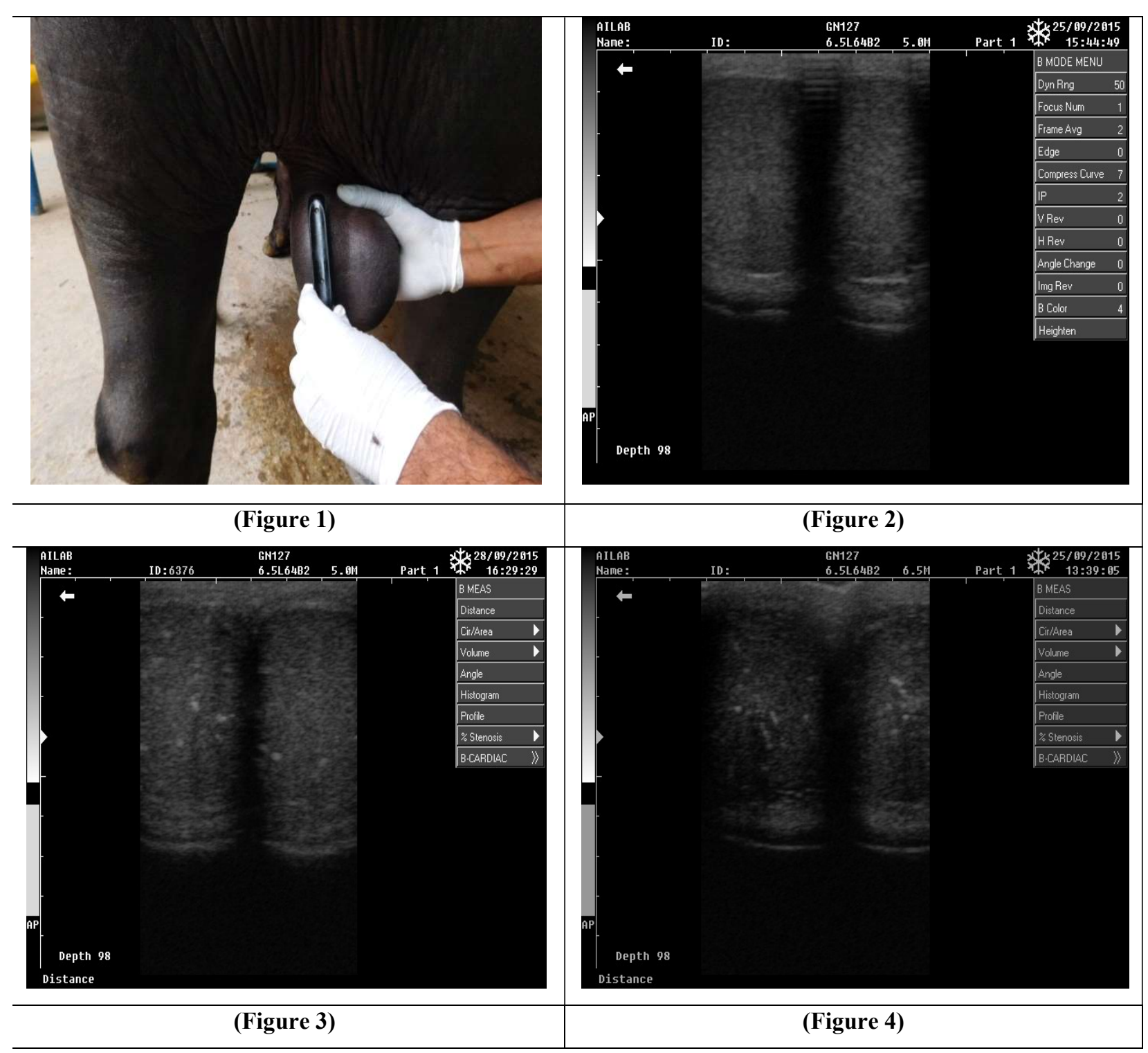

Figure 1. Ultrasonography linear probe placed longitudinally on dorsal surface of testis, Figure 2. Normal testicular parenchyma,

Figure 3 and 4. Testicular parenchyma with shining crystals like structure.

consistency as compared to other groups of soft and firm/hard consistency (Table 1). The resilience was found lower in the testis of soft and firm/hard consistency and higher in moderate consistency. Ashwood (2009) observed that the testes with firm to moderate consistency produced good quality semen as compared to soft in Brahman bulls and bulls with soft testicles with low resilience found to be associated with high percentage of abnormal sperms and low reproductive performance. Testicular consistency was reported to have high positive correlation $(\mathrm{r}=0.59$ to 0.94$)$ with seminal quality, and highly repeatable $(\mathrm{r}=0.95)$ and heritable $(\mathrm{Hz}=0.34)$ trait in Holstein bulls (Foote et al., 1979). In cross bred beef bulls, the reduction in sperm defects was also observed which was associated with an increase in testicular consistency i.e. firmness (Cook et al., 1994). In present study, we also observed deposition of hyperechoic shining crystals like structure in testicular parenchyma of bulls $(n=7)$ during ultrasonographic examination of testis, which having firm/hard testicular consistency and low resilience (Figure. 3 and 4).Whereas, no any deposition of such shining crystals like structure was seen in the testicular parenchyma of soft and moderate consistency and also produce better quality semen (Table 1). The testis becomes hard and losses their resilience may be due to fibrosis in testicular tissues or formation of crystals like structure in testicular parenchyma and leads to degeneration of testicular germinal cell and influence the spermatogenesis. 
Therefore, the quality of semen becomes poor in Murrah bulls which having firm/hard testicular consistency.

\section{Conclusions}

From present study it can be concluded that the bulls having moderate testicular consistency produced better quality semen therefore testicular consistency can also be used as a criterion in routine breeding soundness evaluation for quality semen production.

\section{Acknowledgements}

The authors are thankful to In-charge Artificial Breeding Research Centre of NDRI, Karnal and In-charge officers of different frozen semen stations viz. ICAR-CIRB, GADVASU and HLDB Yamunanagar for allowing us to utilize the data of their breeding bulls. We are highly grateful to Director ICAR-NDRI, Karnal, Haryana, (India) for providing necessary facilities and financial support for carrying out this present work.

\section{References}

Ashwood A (2009) Bull reproductive soundness. Brahaman news 164: 1-6 (http://www.brahman.com.au/technical information)

BAHS (2017) Annual report 2017 Basic Animal Husbandry and Fisheries Statistics. Ministry of Agriculture \& Farmers Welfare, Govt. of India, New Delhi

Cook RB Coulter GH, Kastelic JP (1994) The testicular vascular cone, scrotal thermoregulation, and their relationship to sperm production and seminal quality in beef bulls. Theriogenology 41: 653-671

Foote RH Hahn J and Larson LL (1979) Testicular measurements as predictors of sperm output and semen quality. Proc 3rd Tech Conf A I Reprod 31-35

Kiani FA Yousaf A Zafar MA Nawaz M Akbar Z Sohoo MUR, Magsi AS (2014) Effect of age on physical characteristics of kundhi buffalo bull semen. Int. J. Curr. Microbiol. App Sci 3(11): 445-453

Singh M, Pant HC (2000) Effect of post thaw incubation on semen quality of buffalo bull comparison with cattle. Buffalo bulletin 19(3) 